\title{
Linear Matrix Inequality-based Robust Controller design for Type-1 Diabetes Model $^{\star}$
}

\author{
Péter Szalay, ${ }^{*, * *}$ György Eigner, ${ }^{* * *}$ Levente A. Kovács ${ }^{* * *}$ \\ * Department of Control Engineering and Information Technology, \\ Budapest University of Technology and Economics, Budapest, \\ Hungary, (Tel: +3614634027; e-mail: szalaip@iit.bme.hu) \\ ** Austrian Center for Medical Innovation and Technology (ACMIT), \\ Viktor Kaplan-Straße 2, 2700 Wiener Neustadt-Austria \\ *** Institute of Information Systems, John von Neumann Faculty of \\ Informatics, Obuda University, Budapest, Hungary, (Tel: +361 666 \\ 5585; e-mail: eigner.gyorgy@phd.uni-obuda.hu, \\ kovacs.levente@nik.uni-obuda.hu)
}

\begin{abstract}
This paper investigates the capabilities of a sophisticated robust nonlinear controller designed directly for a widely known and used high-order nonlinear type 1 diabetes (T1DM) model to lessen the dependency from patient compliance and to answer practical requirements such as avoiding hypoglycaemia. The resulting controller can perform adequately in nominal conditions, but expected to keep this performance even in extreme situations, e.g. high carbohydrate intake, rejecting hypoglycaemic episodes.
\end{abstract}

Keywords: Generalized linear systems; Biomedical control; Nonlinear control; Robustness.

\section{INTRODUCTION}

Diabetes mellitus is the dysfunction of the human glucose regulation system that is currently incurable, but treatable. The World Health Organization predicts that the number of patients can soar to 552 million approximately worldwide by 2030, Wild et al. (2004), doubling the diabetes population of 2000 . The treatment of this disease is therefore of paramount importance. Classical therapy of type one diabetes mellitus (T1DM) consists of injections administered by the patient to replace the otherwise impaired production of insulin, which is a hormone of key importance in human metabolism.

The automatic glucose regulation for T1DM patients, the Artificial Pancreas (AP), aims to keep the blood glucose concentration in the normoglycemic range of $70-110$ $\mathrm{mg} / \mathrm{dL}(3.9-6 \mathrm{mmol} / \mathrm{L})$ more effectively than the currently existing therapies without relying on the compliance of the patient. Moreover, it is required to avoid dangerously low glucose levels that could directly endanger the patients' life. It consists of a sensor, an insulin pump and a control algorithm Harvey et al. (2010).

From engineering standpoint this is a rather complicated disturbance rejection problem using a single glucose sen-

\footnotetext{
* P. Szalay is supported by the Austrian Center for Medical Innovation and Technology (ACMIT). L. Kovács is supported by the János Bolyai Research Scholarship of the Hungarian Academy of Sciences. The work is partially supported by the Hungarian National Development Agency GOP-1.1.1.-11-2012-0055 project and by the European Union TÁMOP-4.2.2.A-11/1/KONV-2012-0073 project. The authors say special thanks to Prof. Zoltán Benyó from Budapest University of Technology and Economics.
}

sor, control algorithm and insulin pump, although dualhormone approach also exists Banks et al. (2011). An adequate mathematical model of human metabolism - like the ones presented in Magni et al. (2009) and Wilinska et al. (2010) - is usually rather complicated, where verification and parameter identification alone are challenging tasks Kirchsteiger et al. (2011), while model-based control algorithms must handle nonlinearity and inaccuracy alike. The commercially available continuous glucose measurement (CGM) sensor has significant measurement noise and five minutes sampling time Battelino and Bolinder (2008). Moreover, even when using the most rapid acting insulin analogue in the pump, the time constant of the meal intake having effect on the blood glucose level is shorter due to the use of subcutaneous route. Yet, the research has reached clinical testing phase in the last couple of years Cobelli et al. (2011).

Considering the control algorithm in the AP there were various different approaches, including classical PID controllers Palerm (2011), run-to-run control Zisser et al. (2009), fuzzy logic based control Phillip et al. (2013), model-free soft computing-based control Zarkogianni et al. (2011), exact linearization based nonlinear control Palumbo et al. (2011), $\mathcal{H}_{\infty}$ control Parker et al. (2000) and Model Predictive Control (MPC) Kovatchev et al. (2010), among others.

Although MPC is one of the most effective in individualbased therapies, in practice the controlled process can deviate from the nominal model. Hence, robust methods are required in order to satisfy general requirements (like avoiding hypoglycaemia) under unexpected situations or 
extreme conditions as well. Modern robust control, like $\mathcal{H}_{\infty}$ methodology are predetermined for such cases and regarding the AP problem linear $\mathcal{H}_{\infty}$ robust controllers are gaining popularity Femat et al. (2009), Kovács and Szalay (2012). However, the nonlinearity of the system is difficult to handle for a linear controller alone. Linear Parameter Varying (LPV) controllers could capture the dynamics of the glucose-insulin interaction well Kovács et al. (2011) Pena et al. (2011), making direct nonlinear control possible. The combination of the two approaches have high potential for glucose control Kovács et al. (2013).

This paper investigates whether the capabilities of a robust LPV controller could be extended by additional constraints to answer certain practical issues, such as avoiding hypoglycaemia and high carbohydrate intake. The design will be performed directly on the model which will be presented in Section 2, as well as how the LPV model is defined. Section 3 details each step of the controller design, while simulation results using virtual patient data will be presented in Section 4. The paper is concluded with Section 5, where further research directions will be given as well.

\section{DIABETES MODEL}

The model presented by Wilinska et al. (2010) is described by the following differential equations:

$$
\begin{aligned}
& \dot{C}(t)=-k_{a, i n t} C(t)+\frac{k_{a, i n t}}{V_{G}} Q_{1}(t) \\
& \dot{Q}_{1}(t)=-\left(\frac{F_{01}}{Q_{1}(t)+V_{G}}+x_{1}(t)\right) Q_{1}(t)+k_{12} Q_{2}(t)- \\
& -R_{c l} \max \left\{0, Q_{1}(t)-R_{t h r} V_{G}\right\}-P h y(t)+ \\
& +E G P_{0} \max \left\{0,1-x_{3}(t)\right\}+\min \left\{U_{G, c e i l}, \frac{G_{2}(t)}{t_{\max }}\right\} \\
& \dot{Q}_{2}(t)=x_{1}(t) Q_{1}(t)-\left(k_{12}+x_{2}(t)\right) Q_{2}(t) \\
& \dot{x}_{1}(t)=-k_{b 1} x_{1}(t)+S_{I T} k_{b 1} I(t) \\
& \dot{x}_{2}(t)=-k_{b 2} x_{2}(t)+S_{I D} k_{b 2} I(t) \\
& \dot{x}_{3}(t)=-k_{b 3} x_{3}(t)+S_{I E} k_{b 3} I(t) \\
& \dot{I}_{(}(t)=\frac{k_{a}}{V_{I}} S_{2}(t)-k_{e} I(t) \\
& \dot{S}_{2}(t)=-k_{a} S_{2}(t)+k_{a} S_{1}(t) \\
& \dot{S}_{1}(t)=-k_{a} S_{1}(t)+u(t) \\
& \dot{G}_{2}(t)=\frac{G_{1}(t)-G_{2}(t)}{\max \left\{t_{\max }, \frac{G_{2}(t)}{U_{G, c e i l}}\right\}} \\
& \dot{G}_{1}(t)=-\frac{G_{1}(t)}{\max \left\{t_{\max }, \frac{G_{2}(t)}{U_{G, c e i l}}\right\}}+D(t)
\end{aligned}
$$

where the state variables are: $C(t)$ glucose concentration in the subcutaneous tissue $[\mathrm{mmol} / \mathrm{L}], Q_{1}(t)$ and $Q_{2}(t)$ the masses of glucose in accessible and non-accessible compartments $[\mathrm{mmol}], x_{1}(t), x_{2}(t)$ and $x_{3}(t)$ remote effect of insulin on glucose distribution, disposal and endogenous glucose production respectively [1/min], $I(t)$ insulin concentration in plasma $[\mathrm{mU} / \mathrm{L}], S_{1}(t)$ and $S_{2}(t)$ insulin masses in the accessible and non-accessible compartments [mU], $G_{1}(t)$ and $G_{2}(t)$ glucose masses in the accessible and non-accessible compartments [mmol]. $u(t)$ injected insulin flow of rapid-acting insulin $[\mathrm{mU} / \mathrm{min}]$ is the input of the system, while $D(t)$ amount of ingested carbohydrates $[\mathrm{mmol} / \mathrm{min}]$, and $P h y(t)$ effect of physical activity $[\mathrm{mmol} / \mathrm{min}]$ are considered as disturbances. The following parameters are time-varying with $\pm 5 \%$ deviation: $k_{a, i n t}$, $F_{01}, k_{12}, E G P_{0}, k_{b 1}, k_{b 2}, k_{b 3}, S_{I T}, S_{I D}, S_{I E}, k_{a}$ and $k_{e}$. This is represented by sinusoidal oscillations superimposed on the nominal values with 3 hour period and a randomly generated phase.

The system is complete with a sensor model additionally defined by us, which defines 5 minutes sampling time and white additive measurement noise with $1 \mathrm{mmol}^{2} / \mathrm{L}^{2}$ variance.

The model (1) contains elements such as $F_{01} Q_{1}(t)\left(Q_{1}(t)+\right.$ $\left.V_{G}\right)^{-1}, x_{1}(t) Q_{1}(t)$ and $x_{2}(t) Q_{2}(t)$ which are nonlinear functions of certain state variables. By introducing the notation:

$$
\rho(t)=\left(\begin{array}{c}
\rho_{1}(t) \\
\rho_{2}(t) \\
\rho_{3}(t)
\end{array}\right)=\left(\begin{array}{c}
Q_{1}(t) \\
F_{01}\left(Q_{1}(t)+V_{G}\right)^{-1} \\
Q_{2}(t)
\end{array}\right)
$$

we can capture the model with an LPV system of the following form:

$$
\begin{gathered}
\dot{\mathrm{x}}(t)=\mathbf{A}(\rho(t)) \mathrm{x}(t)+\mathbf{B}(\rho(t)) \mathrm{u}(t) \\
\mathrm{y}(t)=\mathbf{C}(\rho(t)) \mathrm{x}(t)+\mathbf{D}(\rho(t)) \mathrm{u}(t) \\
\mathbf{A}(\rho)=\mathbf{A}_{0}+\prod_{i=1}^{m} \rho_{i}(t) \mathbf{A}_{i} \mathbf{B}(\rho)=\mathbf{B}_{0}+\prod_{i=1}^{m} \rho_{i}(t) \mathbf{B}_{i} \\
\mathbf{C}(\rho)=\mathbf{C}_{0}+\prod_{i=1}^{m} \rho_{i}(t) \mathbf{C}_{i} \mathbf{D}(\rho)=\mathbf{D}_{0}+\prod_{i=1}^{m} \rho_{i}(t) \mathbf{D}_{i}
\end{gathered}
$$

since the chosen scheduling variables are bounded together with their time derivatives as well. State variables $x_{1}(t)$ and $x_{2}(t)$ are also candidates for scheduling variables replacing $Q_{1}(t)$ and $Q_{2}(t)$, which is more advantageous for observer design Szalay et al. (2013).

$\rho_{2}(t)$ is a nonlinear function of $\rho_{1}(t)$. However, as shown in Fig. 1 treating them as independent variables requires the controller to needlessly ensure stability and performance for scheduling variable configurations which would never appear in practice (solid line). There are two possible approaches to overcome these limitations. One would be to give a linear estimation of the function $F_{01}\left(Q_{1}(t)+V_{G}\right)^{-1}$ and hence eliminating $\rho_{2}(t)$ (dotted line). This would simplify the problem greatly by reducing the number of variables during controller synthesis.

On the other hand it introduces further parameter uncertainty to the system. Another possibility is to use a linear $\mathcal{T}$ transformation on the scheduling parameters (4) and the nominal model (5) in order to minimize $\prod_{i=1}^{m} \rho_{i}(t)$ (dashed line). The controller synthesis will be performed on the transformed system and inverse transformation on the resulting controller.

All 3 approaches will be investigated and compared.

$$
\left(\begin{array}{c}
\tilde{\rho}_{1}(t) \\
\tilde{\rho}_{2}(t)
\end{array}\right)=\left[\begin{array}{cc}
\cos (\alpha) & -\sin (\alpha) \\
\sin (\alpha) & \cos (\alpha)
\end{array}\right]\left(\begin{array}{c}
\rho_{1}(t) \\
\rho_{2}(t)
\end{array}\right)-\left(\begin{array}{c}
\rho_{1, \min } \\
\rho_{1, \max }
\end{array}\right)(4)
$$




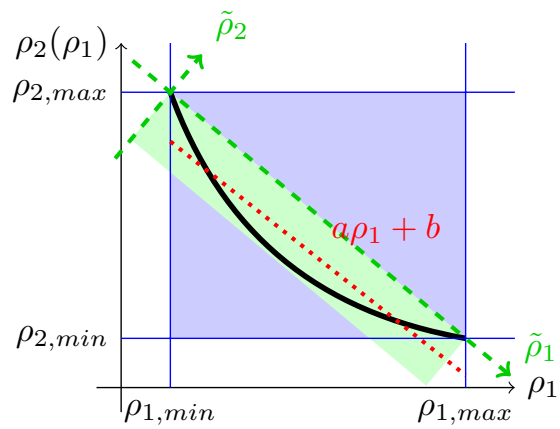

Fig. 1. Different configurations for LPV system. Solid line: classical approach; dashed line: transformed scheduling variables; dotted line: linear approximation.

$$
\begin{aligned}
\mathbf{A}_{0}+\prod_{i=1}^{m} \rho_{i}(t) \mathbf{A}_{i} & =\tilde{\mathbf{A}}_{0}+\prod_{i=1}^{m} \tilde{\rho}_{i}(t) \tilde{\mathbf{A}}_{i} \\
\mathbf{B}_{0}+\prod_{i=1}^{m} \rho_{i}(t) \mathbf{B}_{i} & =\tilde{\mathbf{B}}_{0}+\prod_{i=1}^{m} \tilde{\rho}_{i}(t) \tilde{\mathbf{B}}_{i} \\
\mathbf{C}_{0}+\prod_{i=1}^{m} \rho_{i}(t) \mathbf{C}_{i} & =\tilde{\mathbf{C}}_{0}+\prod_{i=1}^{m} \tilde{\rho}_{i}(t) \tilde{\mathbf{C}}_{i} \\
\mathbf{D}_{0}+\prod_{i=1}^{m} \rho_{i}(t) \mathbf{D}_{i} & =\tilde{\mathbf{D}}_{0}+\prod_{i=1}^{m} \tilde{\rho}_{i}(t) \tilde{\mathbf{D}}_{i}
\end{aligned}
$$

Furthermore, since endogenous glucose production and renal extraction are only present in certain regions of the state space, it is necessary to introduce switching to the controller. Based on Doyle (2012) one can define several different zones, and define a controller for each. Here only 6 controllers will be used based on the value of $Q_{1}(t)$ and $x_{3}(t)$. The two regions defined for the latter are $x_{3}(t) \leq 1$ and $x_{3}(t)>1$ and three for the former as follows:

- The blood glucose concentration is close to the reference $4.9 \mathrm{mmol} / \mathrm{L}: \underline{Q}_{1, r e f} \leq \frac{Q_{1}(t)}{V_{G}}<\bar{Q}_{1, \text { ref }}$. Here, the values $4.5 \mathrm{mmol} / \mathrm{L}$ and $5.5 \mathrm{mmol} / \mathrm{L}$ were used;

- The blood glucose concentration is higher than the reference, but there is no renal extraction: $\bar{Q}_{1, \text { ref }} \leq$ $\frac{Q_{1}(t)}{V_{G}}<R_{t h r}$

- The blood glucose concentration is higher than the threshold of renal clearance: $R_{t h r} \leq \frac{Q_{1}(t)}{V_{G}}$;

To conclude, we can define six nominal LPV models $P_{0, j}, j=1, \ldots, 6$ from (1) for model-based controller design. The differential equations for state variables $G_{1}(t)$ and $G_{2}(t)$ are excluded, and will be used to construct a weighting function capturing worst case meal intake instead.

\section{CONTROLLER DESIGN}

\subsection{Uncertainties}

To design a robust controller it is necessary to examine the uncertainty of the controlled process. There are two main sources of uncertainty: parameter inaccuracy and scheduling parameter inaccuracy. The latter comes from the requirement that for LPV controller $\rho(t)$ must be available for measurement. In this case this is not

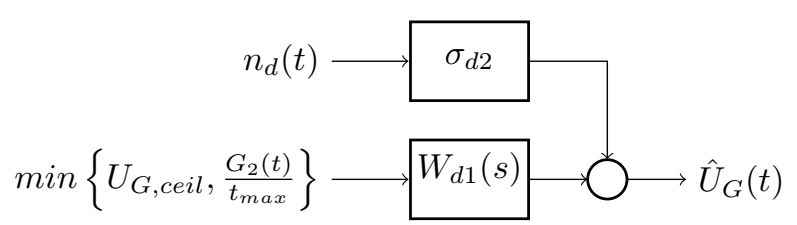

Fig. 2. Glucose flux estimation

satisfied. A form of extended Kalman-filter was used for scheduling parameter and meal intake estimation. To be more specific the following state variables were estimated: $C(t), Q_{1}(t), Q_{2}(t), x_{3}(t), G_{2}(t)$. Then, we assumed that the estimated scheduling parameters $\hat{\rho}(t)$ were the correct ones, it is the system has parameter inaccuracy. The resulting error was divided into multiplicative inaccuracy and additive error:

$$
\begin{gathered}
\left(\begin{array}{c}
\rho_{1}(t) x_{1}(t) \\
\rho_{2}(t) Q_{1}(t) \\
\rho_{3}(t) Q_{2}(t)
\end{array}\right)=\left(\begin{array}{c}
\left(\hat{\rho}_{1}(t)\left(1+\Delta_{1}\right)+\tilde{\rho}_{1}(t)\right) x_{1}(t) \\
\left(\hat{\rho}_{2}(t)\left(1+\Delta_{2}\right)+\tilde{\rho}_{2}(t)\right) \\
\left(\begin{array}{c}
\left.\hat{\rho}_{3}(t)\left(1+\Delta_{3}\right)+\tilde{\rho}_{3}(t)\right) \\
Q_{1}(t)
\end{array}\right) Q_{2}(t)
\end{array}\right)= \\
\left(\begin{array}{c}
\hat{\rho}_{1}(t)\left(1+\Delta_{1}\right) x_{1}(t)+d_{\rho, 1}(t) \\
\hat{\rho}_{2}(t)\left(1+\Delta_{2}\right) Q_{1}(t)+d_{\rho, 2}(t) \\
\hat{\rho}_{3}(t)\left(1+\Delta_{3}\right) Q_{2}(t)+d_{\rho, 3}(t)
\end{array}\right)
\end{gathered}
$$

where $\Delta \rho_{i}$ are the multiplicative inaccuracies of the scheduling parameters, $\tilde{\rho}_{i}(t)$ are additive errors which multiplied with the respective state variables define additional $d_{\rho, i}(t)$ disturbance inputs for the systems $(i=1,2,3)$. Using this setup we can apply LPV control for this system with the cost of increased inaccuracy and disturbances. Moreover, it was found that it is more advantageous to make the multiplicative inaccuracies a function of the respective scheduling variables: $\Delta \rho_{i}\left(\rho_{i}(t)\right)$.

Since the estimation of the glucose flux from the gut $\hat{U}_{G}(t)=\frac{\hat{G}_{2}(t)}{t_{\max }}$ is available as well, one can define a SISO transfer function $W_{d 1}(s)$ and a zero mean additive noise with variance $\sigma_{d 2}^{2}$ to define the connection between the estimated and the real value (Fig. 2).

Finally, the estimated output $\hat{C}(t)$ was used for feedback to the controller instead of the real measurement. The estimation error can be seen as a virtual measurement noise, which has usually a smaller variance $\left(\sigma_{C}^{2}\right)$ than the actual measurement noise.

The values of $\Delta \rho_{i}\left(\rho_{i}(t)\right), d_{\rho, i}(t), W_{d 1}(s), \sigma_{d 2}$ and $\sigma_{C}$ were determined from 500 Monte Carlo simulations for each virtual patien using randomized meal intakes, physical activity, insulin injections, parameter inaccuracies and measurement noise.

Based on sensitivity analysis a single output multiplicative uncertainty weighting function $\left(W_{o u t, j}(s)\right)$ was constructed for each controller $(j=1, \ldots, 6)$ of each virtual patient. They have the following form:

$$
W_{\text {out }, j}(s)=\frac{s+z_{j}}{s+p_{j}}
$$

$W_{\text {out }, j}(s)$ represents $100 \%$ uncertainty for higher frequencies, and $100 \frac{z_{j}}{p_{j}} \%$ for lower frequencies.

It must be stressed, that the original model (1) has been used directly for controller synthesis. Even though certain 




Fig. 3. Complete model for controller design.

state variables have been discarded, it was only necessary if they added poles to the model with time constants smaller than the sensor sampling time. The resulting deviation from (1) was included in $W_{\text {out }, j}(s)$.

\subsection{Weighting functions}

To design a robust controller, there are several other weighting functions to be defined. $W_{\text {meal }}(s)$ describes worst case meal intake, $W_{u}=\frac{1}{u_{\max }}$ represents constraints of maximum insulin input $\left(u_{\max }\right)$ and $W_{n}=\sigma_{C}$ captures measurement noise.

$$
W_{\text {meal }}(s)=U_{G, \text { ceil }} \frac{t_{\text {max }}^{2}}{\left(s+t_{\max }\right)^{2}}
$$

Performance weighting function $W_{\text {per }}(s)$ is used to specify the requirements for how much deviation from the 4.9 $\mathrm{mmol} / \mathrm{L}$ reference glucose concentration is tolerated. The following function (9) allows 10 times larger errors for higher frequencies than for lower frequencies:

$$
W_{\text {per }}(s)=\frac{30 s+1}{300 s+1}
$$

It is possible to have intergral or two degree-of-freedom controller by adding specific weighting functions.

The complete system is displayed in Fig. 3.

\subsection{Controller synthesis}

Based on Zhou (1996), Ghaoui and Niculescu (2000) and Scherer and Weiland (2004) for an LPV system of the form (10) it is possible to find an LPV controller of the form (11) and a constant $\gamma$, so that the closed-loop system is stable and the transfer from the input $d(t)$ to the output $z(t)$ has an $\mathcal{H}_{\infty}$ norm smaller or equal than $\gamma$.

$$
\begin{aligned}
\dot{\mathrm{x}}(t) & =\mathbf{A}(\rho(t)) \mathrm{x}(t)+\mathbf{B}_{1}(\rho(t)) \mathrm{d}(t)+\mathbf{B}_{2} \mathrm{u}(t) \\
\mathrm{z}(t) & =\mathbf{C}_{1}(\rho(t)) \mathrm{x}(t)+\mathbf{D}_{11}(\rho(t)) \mathrm{d}(t)+\mathbf{D}_{12} \mathrm{u}(t) \\
\mathrm{y}(t) & =\mathbf{C}_{2} \mathrm{x}(t)+\mathbf{D}_{21} \mathrm{~d}(t) \\
& \dot{\mathrm{x}}_{c}(t)=\mathbf{A}_{c}(\rho(t)) \mathrm{x}_{c}(t)+\mathbf{B}_{c}(\rho(t)) \mathrm{y}(t) \\
& \mathrm{u}(t)=\mathbf{C}_{c}(\rho(t)) \mathrm{x}_{c}(t)+\mathbf{D}_{c}(\rho(t)) \mathrm{y}(t)
\end{aligned}
$$

The controller synthesis is performed by solving a corresponding linear matrix inequality (LMI) while minimizing $\gamma$. Although there are various alternative techniques, the greatest advantage of this method would be that it defines a convex constraint on the sought variables. Furthermore, a finite set of LMIs $\mathbf{M}_{1}(\mathrm{x})<0, \ldots, \mathbf{M}_{n}(\mathrm{x})<0$ can

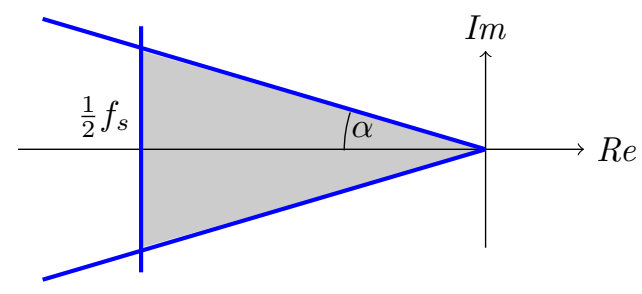

Fig. 4. Stability region for controller design

be combined into a single LMI (12). More precisely, $\mathbf{M}_{1}(\mathrm{x})<0, \ldots, \mathbf{M}_{n}(\mathrm{x})<0$ if and only if

$$
\left(\begin{array}{ccc}
\mathbf{M}_{1}(\mathrm{x}) & & \\
& \ddots & \\
& & \mathbf{M}_{n}(\mathrm{x})
\end{array}\right)<0 .
$$

This means we can create hybrid controllers which can satisfy more than one requirement for norms. In this particular case, we had two separate LMIs. One was to ensure the robust stability of the system and that the performance criteria for control signal will be met. From all disturbance inputs to the two outputs $\Delta_{\text {out }}$ and $z_{u}(t)$ the $\mathcal{H}_{\infty}$ norm of the system must be less than 1 . The other LMI was to minimize the $\mathcal{H}_{\infty}$ norm of the transfer function from all outputs to the output of the performance weighting function $z_{e}(t)$.

Needless to say it is possible to have requirements based on different norms, such as $\mathcal{H}_{2}$ or $\mathcal{L}_{1}$, but this is not the scope of this paper.

There are several other important constraints we can impose on the controller which can be captured with LMIs. One of these which have high importance in blood glucose control define stability region for the poles of the closed-loop system. The model is usually defined in continuous time while the controller must be implemented in discrete time. Hence, there cannot be poles faster than the sampling time of the glucose measurement sensor. Moreover, since negative control signal has no physical meaning in this application, it is undesirable to have oscillatory transients. Therefore, instead of being satisfied with closed-loop poles having negative real value, we define a stability region as shown in Fig. 4.

In this case the constraints for the poles $p$ of the closedloop system are $0>\operatorname{Re}\{p\}>0.1$ and $1>\frac{\operatorname{Im}\{p\}}{\operatorname{Re}\{p\}}$.

\section{RESULTS}

To show the capabilities of the proposed robust controller simulations were conducted using the parameter sets of 6 virtual patients presented in Wilinska et al. (2010). For each patient three LPV controllers were created using different settings for the scheduling variables (Fig. 1). Simulations were conducted using three different protocols:

(1) 2 days simulation with $150 \mathrm{~g}$ of carbohydrate (CHO) intake per day (classical T1DM patient protocol). The considered meal intake consists of a $35 \mathrm{~g} \mathrm{CHO}$ breakfast at 8:30, a $65 \mathrm{~g} \mathrm{CHO}$ lunch at 13:00, and 50 g CHO dinner at 19:00.

(2) 2 days simulation based on Magni et al. (2008) meal intake protocol. It consists of a $45 \mathrm{~g}$ CHO breakfast 


\begin{tabular}{|c|c|c|c|}
\cline { 2 - 4 } \multicolumn{1}{c|}{} & $\begin{array}{l}\text { Chance of } \\
\text { occurence }\end{array}$ & Amount $[g]$ & Time [hour $]$ \\
\hline Breakfast & $100 \%$ & $40-60$ & $6: 00-10: 00$ \\
\hline Snack 1 & $50 \%$ & $5-25$ & $8: 00-11: 00$ \\
\hline Lunch & $100 \%$ & $70-110$ & $11: 00-15: 00$ \\
\hline Snack 2 & $50 \%$ & $5-25$ & $15: 00-18: 00$ \\
\hline Dinner & $100 \%$ & $55-75$ & $18: 00-22: 00$ \\
\hline Snack 3 & $50 \%$ & $5-15$ & $22: 00-0: 00$ \\
\hline
\end{tabular}

Table 1. High carbohydrate intake simulation parameters

\begin{tabular}{|c|c|c|c|}
\hline LPV type & Classic & Simplified & Transformed \\
\hline \hline $\begin{array}{c}\text { Hypo } \\
<3.9 \mathrm{mmol} / \mathrm{L}\end{array}$ & $0.00 \%$ & $0.00 \%$ & $0.00 \%$ \\
\hline $\begin{array}{c}\text { Normal } \\
3.9-6 \mathrm{mmol} / \mathrm{L}\end{array}$ & $27.62 \%$ & $28.97 \%$ & $19.63 \%$ \\
\hline $\begin{array}{c}\text { Mild hyper } \\
6-7.8 \mathrm{mmol} / \mathrm{L}\end{array}$ & $14.45 \%$ & $15.67 \%$ & $22.13 \%$ \\
\hline $\begin{array}{c}\mathrm{Hyper} \\
6-11 \mathrm{mmol} / \mathrm{L}\end{array}$ & $41.64 \%$ & $44.88 \%$ & $63.45 \%$ \\
\hline $\begin{array}{c}\text { Severe hyper } \\
>11.1 \mathrm{mmol} / \mathrm{L}\end{array}$ & $30.73 \%$ & $26.15 \%$ & $16.92 \%$ \\
\hline
\end{tabular}

Table 2. Simulation results for high carbohydrate intake scenario.

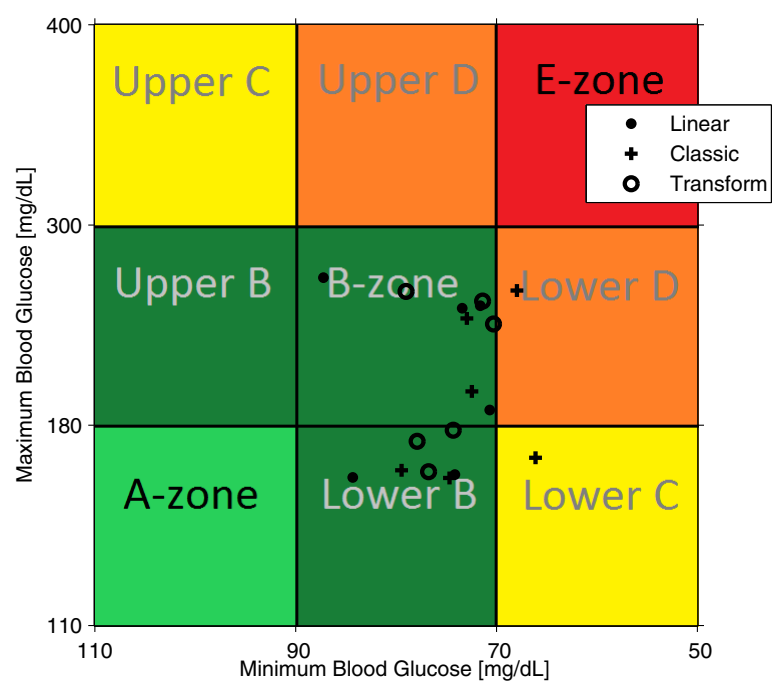

Fig. 5. CVGA of all LPV controllers for $150 \mathrm{~g}$ of daily carbohydrate intake

at 9:30, a $75 \mathrm{~g} \mathrm{CHO}$ lunch at 13:30, and $85 \mathrm{~g} \mathrm{CHO}$ dinner at 19:30.

(3) 2 days simulation with irregular meal timing and unusually high CHO intake ( Table 1).

For all simulations the measurement noise and the parameter inaccuracies were randomized. An example for simulation is shown in Fig. 7. The results for the first two meal scenario are presented for all virtual patients and controller types in Fig. 5 and Fig. 6 using control variability grid analysis (CVGA) similarly to Magni et al. (2008). For larger meal intake (scenarion 3) the results are summarized in Table 2 based on 50 simulations for each virtual patient. The values given are the average time spent in various blood glucose ranges in percentage of the whole simulation.

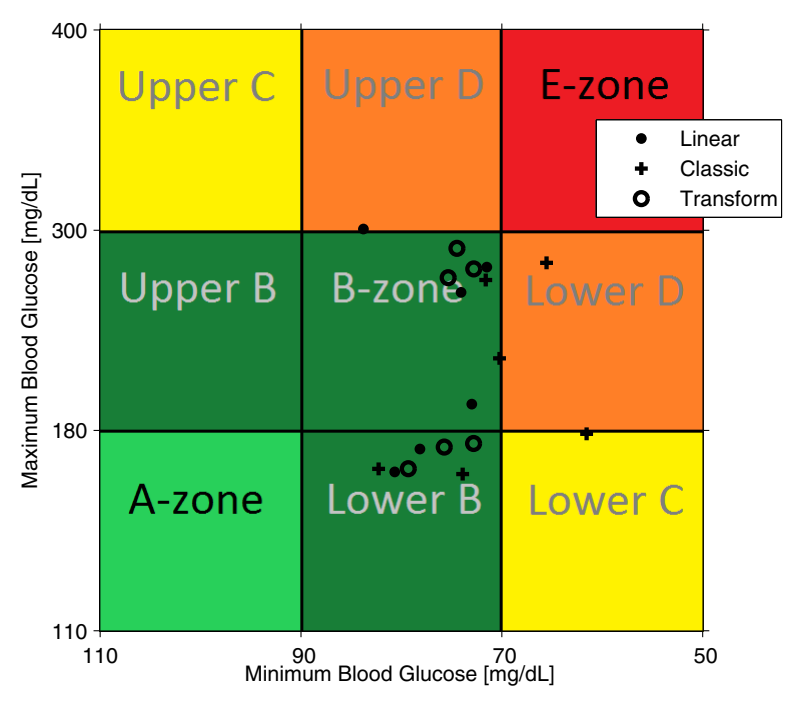

Fig. 6. CVGA of all LPV controllers for (2) meal intake protocol
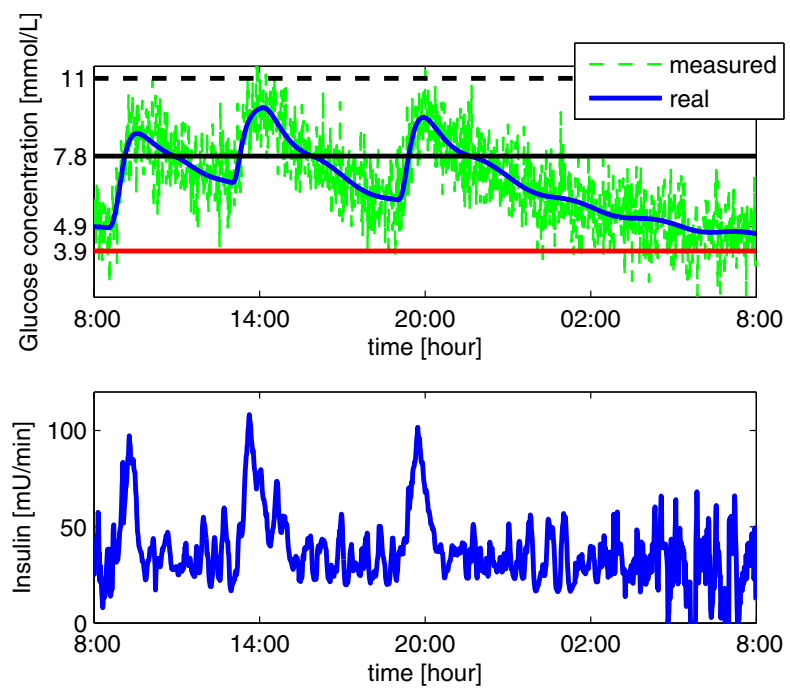

Fig. 7. Example for virtual patient simulation

It can be concluded, that based on the acquired results in case of linear approximation of the second scheduling variable or transformation of the first two scheduling variables can avoid hypoglycaemia. The latter can maintain all patients in the lower B and B zones, which is the requirement for a robust controller. Furthermore, these two controllers can adequately compensate for higher meal intake as well. However, more effective disturbance rejection is possible considering non-randomized meal protocols and a strict regime, but here, the scope of the paper was to demonstrate the robust characteristics of the designed nonlinear controllers.

\section{CONCLUSION}

The design process of three different robust LPV controllers for blood glucose control in case of T1DM has been presented. It can be concluded that robustness is achieved even in extreme conditions demonstrating the effectiveness of the methodology and avoiding hypoglycaemia. Moreover, the capabilities of the designed con- 
trollers were demonstrated on the real nonlinear system (without reduction or linearization). Further work will include the investigation whether introducing integrator or two degree-of-freedom controller structure would improve the results. Moreover, the performance requirements could be defined in $\mathcal{H}_{2}$ or $\mathcal{L}_{1}$ norm as well. Different T1DM models, although represent the same process, have different properties, advantages and disadvantages from controller design point of view. More sophisticated sensor models and different filtering methods can potentially bring further improvements or address different practical issues. Finally, these controllers can be combined with MPC techniques to utilize the benefits of sharply tuned individualized therapy with robust guarantees.

\section{ACKNOWLEDGEMENTS}

The authors say special thanks to the Hungarian Artificial Pancreas working group's insulin pump centers for the real data provided to validate the nonlinear model-based T1DM robust control algorithm as well as for Prof. Dr. László Barkai, president of the Hungarian Diabetes Association for his continuous support. Moreover, special thanks are addressed to Dr. Roman Hovorka and Dr. Malgorzata E Wilinska for the Cambridge "Simulator Educational" of type 1 diabetes.

\section{REFERENCES}

Banks, D., Morris, C., Omeru, J., Wei, W., Georgiou, P., and Toumazou, C. (2011). A CMOS pancreatic islet of langerhans for automatic glycemic regulation. In Proc. of IEEE BioCAS Conference, 261-264.

Battelino, T. and Bolinder, J. (2008). Clinical use of realtime continuous glucose monitoring. Curr Diabetes Rev, 4, 218-222.

Cobelli, C., Renard, E., and Kovatchev, B. (2011). Artificial pancreas: Past, present and future. Diabetes, 60(11), 2672-2682.

Doyle, F. (2012). Zone model predictive control of an artificial pancreas. In Proc. of 10th WCICA Conference, Beijing, China, 8-9.

Femat, R., Ruiz-Velazquez, E., and Quiroz, G. (2009). Weighting restriction for intravenous insulin delivery on T1DM patient via $\mathcal{H}_{\infty}$ control. IEEE T Autom Sci Eng, 6(2), 239-247.

Ghaoui, L. and Niculescu, S. (2000). Advances in Linear Matrix Inequality Methods in Control. SIAM, Philadelphia, USA.

Harvey, R., Wang, Y., Grossman, B., Percival, M., Bevier, W., Finan, D., Zisser, H., Seborg, D., Jovanovic, L., Doyle, F., and Dassau, E. (2010). Quest for the artificial pancreas. IEEE Eng Med Biol, 29(2), 53-62.

Kirchsteiger, H., Pölzer, S., Johansson, R., Renard, E., and del Re, L. (2011). Direct continuous time system identification of MISO transfer function models applied to type 1 diabetes. In Proc. of 50th IEEE CDC-ECC Conference, Orlando, USA, 5176-5181.

Kovatchev, B., Cobelli, C., and Renard, E. (2010). Multinational study of subcutaneous model-predictive closedloop control in type 1 diabetes: summary of the results. J Diab Sci Techn, 4, 1374-1381.

Kovács, L., Benyó, B., Bokor, J., and Benyó, Z. (2011). Induced $\mathcal{L}_{2}$-norm minimization of glucose-insulin system for type I diabetic patients. Comp Meth Progr Biomed, 102(2), 105-118.

Kovács, L. and Szalay, P. (2012). Possibilities and boundaries of $\mathcal{H}_{\infty}$ control in type 1 diabetes. In Proc. 8th IFAC Symp. Biological and Medical Systems, 61-66.

Kovács, L., Szalay, P., Almássy, Z., and Barkai, L. (2013). Applicability results of a nonlinear model-based robust blood glucose control algorithm. J Diab Sci Techn, 7(3), 708-716.

Magni, L., Raimondo, D.M., Man, C.D., Nicolao, G.D., Kovatchev, B., and Cobelli, C. (2009). Model predictive control of glucose concentration in type 1 diabetic patients: An in silico trial. Biomed Sign Proc Contr, 338-346.

Magni, L., Raimondo, D., Man, C.D., Breton, M., Patek, S., Nicolao, G.D., Cobelli, C., and Kovatchev, B. (2008). Evaluating the efficacy of closed-loop glucose regulation via control-variability grid analysis. J Diab Sci Techn, 2(4), 630-635.

Palerm, C. (2011). Physiologic insulin delivery with insulin feedback: A control systems perspective. Comp Meth Progr Biomed, 102(2), 130-137.

Palumbo, P., Pizzichelli, G., Panunzi, S., Pepe, P., and Gaetano, A.D. (2011). Tests on a virtual patient for an observer-based, closed-loop control of plasma glycemia. In Proc. of 50th IEEE CDC-ECC Conference, Orlando, USA, 6936-6941.

Parker, R., Doyle, F., Ward, J., and Peppas, N. (2000). Robust $\mathcal{H}_{\infty}$ glucose control in diabetes using a physiological model. AIChE, 46(12), 2537-2549.

Pena, R.S., Ghersin, A., and Bianchi, F. (2011). Timevarying procedures for insulin-dependent diabetes mellitus control. J Electr Comp Eng, 2011, 1-10.

Phillip, M., Battelino, T., Atlas, E., Kordonouri, O., Bratina, N., Miller, S., Biester, T., Stefanija, M., Muller, I., Nimri, R., and Danne, T. (2013). Nocturnal glucose control with an artificial pancreas at a diabetes camp. $N$ Engl J Med, 368(9), 824-833.

Scherer, C. and Weiland, S. (2004). Linear Matrix Inequalities in Control. Lecture Notes, Delft Center for Systems and Control, Delft, The Netherlands.

Szalay, P., Eigner, G., Kozlovszky, M., Rudas, I., and Kovács, L. (2013). The significance of LPV modeling of a widely used T1DM model. In Proc. of 35th IEEE EMBC, Osaka, Japan, 3531-3534.

Wild, S., Roglic, G., Green, A., Sicree, R., and King, H. (2004). Global prevalence of diabetes. estimates for the year 2000 and projections for 2030. Diab Care, 27(5), 1047-1053.

Wilinska, M., Chassin, L., Acerini, C., Allen, J., Dunger, D., and Hovorka, R. (2010). Simulation environment to evaluate closed-loop insulin delivery systems in type 1 diabetes. J Diab Sci Techn, 4(1), 132-144.

Zarkogianni, K., Vazeou, A., Mougiakakou, S., Prountzou, A., and Nikita, K. (2011). An insulin infusion advisory system based on autotuning nonlinear model-predictive control. IEEE J Biomed Eng, 58(9), 2467-2477.

Zhou, K. (1996). Robust and Optimal Control. Prentice Hall, New Jersey.

Zisser, H., Palerm, C., Bevier, W., III, F.D., and Jovanovic, L. (2009). Clinical update on optimal prandial insulin dosing using a refined run-to-run control algorithm. J Diab Sci Techn, 3, 487-491. 